\title{
Giant magnetocapacitance of strained ferroelectric-ferromagnetic hybrids
}

\author{
N. A. Pertsev, ${ }^{1,2}$ S. Prokhorenko, ${ }^{2}$ and B. Dkhil ${ }^{2}$ \\ ${ }^{1}$ A. F. Ioffe Physico-Technical Institute, Russian Academy of Sciences, 194021 St. Petersburg, Russia \\ ${ }^{2}$ Laboratoire Structures, Propriétés et Modélisation des Solides, UMR CNRS - École Centrale Paris, 92295 Châtenay-Malabry, France
}

(Received 27 December 2011; revised manuscript received 20 March 2012; published 27 April 2012)

\begin{abstract}
We report a theoretical calculation of the strain-mediated magnetocapacitance of ferroelectric-ferromagnetic heterostructures. Our nonlinear theory predicts that this magnetocapacitance strongly depends on the strain state of a ferroelectric constituent. For multiferroic hybrids comprising a $\mathrm{BaTiO}_{3}$ or $\mathrm{Pb}\left(\mathrm{Zr}_{0.5} \mathrm{Ti}_{0.5}\right) \mathrm{O}_{3}$ film and a FeBSiC, Terfenol-D, or FeGa substrate, the magnetocapacitive coefficient generally has a giant value $\sim 10^{-4}$ $\mathrm{Oe}^{-1}$. Remarkably, its magnitude further increases drastically near the strain-induced phase transition at which the out-of-plane polarization appears in the ferroelectric film. As a result, the magnetocapacitance of hybrids including Terfenol-D may exceed $100 \%$ already at the magnetic field of about 600 Oe. These theoretical results provide guidelines for the fabrication of multiferroic heterostructures exhibiting a strong magnetodielectric effect.
\end{abstract}

DOI: 10.1103/PhysRevB.85.134111

\section{INTRODUCTION}

The interplay between magnetic and electric phenomena in solids is currently among the hottest topics in condensedmatter physics. ${ }^{1-4}$ This interplay manifests itself either in a dependence of electric properties on an applied magnetic field or in electric-field-induced changes in magnetic properties, which may be termed the direct and converse magnetoelectric (ME) effects, respectively. ${ }^{5}$ Importantly, such ME effects are not only of high fundamental interest, but also of great practical importance. Indeed, the direct and converse ME effects have many potential applications in electronic and spintronic devices, such as room-temperature magnetic-field sensors and electric current probes, ${ }^{6}$ microwave filters, ${ }^{7,8} \mathrm{ME}$ recording read heads, ${ }^{9}$ electric-write magnetic memories, ${ }^{10,11}$ and energy-harvesting devices. ${ }^{12}$

The experimental studies show that the ME responses of multiferroic composites greatly exceed those of singlephase magnetoelectrics. ${ }^{4}$ Recently, the focus of these studies has shifted from bulk composites to multiferroic nanostructures, ${ }^{4,13}$ which are more suitable for microelectronics and may display even stronger ME responses. However, such nanostructures are usually grown on thick passive substrates, ${ }^{13-17}$ which strongly reduce the strain-mediated ME response by suppressing field-induced deformations, especially in heterostructures with the 2-2 connectivity. Fortunately, this drawback can be overcome by using active magnetostrictive ${ }^{18,19}$ or piezoelectric ${ }^{20-23}$ substrates to fabricate multiferroic hybrids designed to display high direct or converse ME effect mediated by lattice strains.

The direct ME effect is usually quantified in terms of the polarization and voltage coefficients, which define the change $\delta \mathbf{P}$ in electric polarization and the associated output voltage $\delta V$ induced by an applied magnetic field $\delta \mathbf{H}$. Since the latter affects the dielectric properties of magnetoelectrics as well, additional important information on the degree of ME coupling is provided by the magnetodielectric coefficients. Importantly, these coefficients can be easily evaluated experimentally by measuring the dependence of capacitance $C$ on magnetic-field intensity [so-called magnetocapacitance (MC)]. As a matter of fact, the measurements of MC are widely used for the characterization of multiferroic heterostructures ${ }^{14-17}$ It should be noted, however, that in some cases the MC effects can
PACS number(s): 75.85.+t, 77.55.fe, 77.55.fg, 77.84.Lf

be complete artifacts as any magnetoresistive material that also has a Maxwell-Wagner space charge will display a magnetodielectric effect. ${ }^{24,25}$

Although the strain-mediated ME polarization and voltage responses of multiferroic composites and nanostructures were already studied theoretically in many papers, ${ }^{19,26-30}$ the calculations of their intrinsic magnetocapacitance were still lacking. Here, we calculate the strain-mediated MC of multiferroic hybrids with the aid of the nonlinear thermodynamic approach used previously to predict ME polarization and voltage coefficients. ${ }^{19,29,30}$ In order to determine the upper bound of the magnetodielectric effect, we consider the optimum case of a thin ferroelectric film coupled to a thick ferromagnetic substrate. Restricting our analysis to a conventional parallelplate ferroelectric capacitor, we calculate sensitivities of the film out-of-plane permittivity to in-plane lattice strains, which govern the strain-mediated MC. Then, taking into account the substrate piezomagnetic coefficients, we evaluate $\mathrm{MC}$ for hybrids comprising (001)-oriented $\mathrm{BaTiO}_{3}$ and $\mathrm{Pb}\left(\mathrm{Zr}_{0.5} \mathrm{Ti}_{0.5}\right) \mathrm{O}_{3}$ [lead zirconate titanate (PZT) 50/50] films coupled to FeGa, Terfenol-D, and FeBSiC substrates.

\section{STRAIN-MEDIATED MAGNETOCAPACITANCE}

The generally accepted definition of $\mathrm{MC}$ reads $\mathrm{MC}=$ $[C(\delta \mathbf{H})-C(0)] / C(0)$. Since the capacitance $C$ depends on the permittivity tensor $\varepsilon_{i j}$ of involved dielectric material, the basic physical quantities defining $\mathrm{MC}$ are the magnetodielectric coefficients $\alpha_{i j k}^{\varepsilon}=\partial \varepsilon_{i j} / \partial H_{k}$. For the strain-mediated ME effect in a film-substrate hybrid, these coefficients can be calculated as

$$
\alpha_{i j k}^{\varepsilon}=\frac{\partial \varepsilon_{i j}}{\partial u_{11}} \frac{\partial u_{11}^{\mathrm{s}}}{\partial H_{k}}+\frac{\partial \varepsilon_{i j}}{\partial u_{22}} \frac{\partial u_{22}^{\mathrm{s}}}{\partial H_{k}}+\frac{\partial \varepsilon_{i j}}{\partial u_{12}} \frac{\partial u_{12}^{\mathrm{s}}}{\partial H_{k}},
$$

where $u_{\alpha \beta}$ and $u_{\alpha \beta}^{\mathrm{s}}(\alpha, \beta=1,2)$ are the in-plane film strains and substrate deformations, respectively, and the perfect mechanical coupling at the film/substrate interface is assumed $\left(\delta u_{\alpha \beta}=\right.$ $\left.\delta u_{\alpha \beta}^{\mathrm{s}}\right)$. Since the deformation response of a ferromagnetic material to the weak measuring magnetic field $\delta \mathbf{H}$ becomes linear under sufficient bias field $\mathbf{H}$, the second factors in Eq. (1) can be replaced by the substrate field-dependent piezomagnetic coefficients $d_{j \alpha \beta}^{\mathrm{m}}=\partial u_{\alpha \beta}^{\mathrm{s}} / \partial H_{j}$. It should be emphasized that 


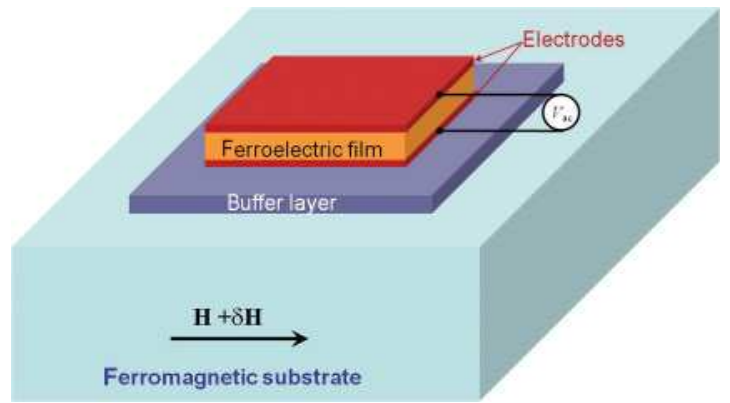

FIG. 1. (Color online) Schematic of a multiferroic hybrid comprising a ferroelectric parallel-plate capacitor and a thick ferromagnetic substrate serving as a magnetostrictive actuator. The heterostructure may incorporate a suitable buffer layer ensuring the growth of a single-crystalline or highly textured ferroelectric film on the bottom electrode.

these quantities characterize the local deformation response of a substrate region beneath the ferroelectric film. Therefore, they generally depend on the substrate geometry. Indeed, when the magnetic field is not parallel to the film/substrate interface, the demagnetizing field evidently makes $d_{3 \alpha \beta}^{\mathrm{m}}$ smaller than the piezomagnetic coefficients measured in an ideal experiment where the internal magnetic field is equal to the applied one.

Let us focus now on the parallel-plate capacitor geometry (Fig. 1), where only the out-of-plane film permittivity $\varepsilon_{33}$ is important. Introducing the strain sensitivities $S_{\alpha \beta}^{\varepsilon}$ of the permittivity $\varepsilon_{33}$ via the relation $S_{\alpha \beta}^{\varepsilon}=\partial \ln \varepsilon_{33} / \partial u_{\alpha \beta}$, we obtain

$$
\begin{aligned}
\mathrm{MC} & =\frac{\varepsilon_{33}(\delta \mathbf{H})-\varepsilon_{33}(0)}{\varepsilon_{33}(0)} \\
& \approx \sum_{j=1}^{3}\left(S_{11}^{\varepsilon} d_{j 11}^{\mathrm{m}}+S_{12}^{\varepsilon} d_{j 12}^{\mathrm{m}}+S_{22}^{\varepsilon} d_{j 22}^{\mathrm{m}}\right) \delta H_{j}
\end{aligned}
$$

Equation (2) is valid for weak fields $\delta \mathbf{H} \ll \mathbf{H}$ that do not change the initial piezomagnetic coefficients and strain sensitivities significantly. The sensitivities $S_{\alpha \beta}^{\varepsilon}$ can be calculated using the nonlinear thermodynamic theory of ferroelectric thin films. ${ }^{31,32}$ (It should be noted that the linear theory does not predict any variation in film permittivity with strain.) In similarity with the film spontaneous polarization $\mathbf{P}$ and permittivity, which strongly depend on misfit strains in the epitaxial system, ${ }^{30,31,33} S_{\alpha \beta}^{\varepsilon}$ are functions of the in-plane lattice strains $u_{\alpha \beta}$ existing in the ferroelectric film prior to the MC measurements. Strictly speaking, these strains are given by the sum of initial strains $u_{\alpha \beta}^{0}$ in the heterostructure and an additional contribution appearing in the bias field $\mathbf{H}$, which in our case may be set equal to the field-induced substrate deformations: $u_{\alpha \beta}=u_{\alpha \beta}^{0}+u_{\alpha \beta}^{s}(\mathbf{H})$. However, usually, $u_{\alpha \beta}^{s}(\mathbf{H})$ are very small so that their contribution to the total in-plane strains may be neglected in a good approximation. ${ }^{30}$

When the film stabilizes in the tetragonal $c$ phase $\left(P_{1}=\right.$ $P_{2}=0, P_{3} \neq 0$ ), the strain sensitivities $S_{\alpha \beta}^{\varepsilon}$ can be calculated analytically. The inspection of the general expression for the film thermodynamic potential $\tilde{G}$ derived in Ref. 32 immediately demonstrates that $S_{11}^{\varepsilon}=S_{22}^{\varepsilon}$ and $S_{12}^{\varepsilon}=0$. Hence, Eq. (2) reduces to $\mathrm{MC}=S_{11}^{\varepsilon} \sum_{j=1}^{3}\left(d_{j 11}^{\mathrm{m}}+d_{j 22}^{\mathrm{m}}\right) \delta H_{j}$ so that magnetocapacitance depends on the sums of substrate piezomagnetic coefficients in this case. The differentiation of $\tilde{G}$ further yields

$$
\begin{aligned}
S_{11}^{\varepsilon}= & S_{22}^{\varepsilon}=-\frac{4 Q_{12}}{\left(s_{11}+s_{12}\right)} \\
& \times\left(1+\frac{3 a_{111} P_{3}^{2}+12 a_{1111} P_{3}^{4}}{a_{33}^{*}+3 a_{111} P_{3}^{2}+6 a_{1111} P_{3}^{4}}\right) \eta_{33},
\end{aligned}
$$

where $\eta_{33}=\left(2 a_{3}^{*}+12 a_{33}^{*} P_{3}^{2}+30 a_{111} P_{3}^{4}+56 a_{1111} P_{3}^{6}\right)^{-1}$ is the out-of-plane dielectric susceptibility of the $c$ phase calculated in the $P^{8}$ approximation, whereas $a_{3}^{*}=a_{1}-$ $Q_{12}\left(u_{11}+u_{22}\right) /\left(s_{11}+s_{12}\right)$ and $a_{33}^{*}=a_{11}+Q_{12}^{2} /\left(s_{11}+s_{12}\right)$ are the renormalized thermodynamic coefficients $\left(a_{1}, a_{11}, a_{111}\right.$, and $a_{1111}$ are the dielectric stiffness and higher-order stiffness coefficients at constant stress, $Q_{l n}$ are the electrostrictive constants, and $s_{l n}$ are the film elastic compliances at a constant polarization). Remarkably, the sensitivities $S_{11}^{\varepsilon}$ and $S_{22}^{\varepsilon}$ are directly proportional to the electrostrictive constant $Q_{12}$ and scale linearly with the film susceptibility $\eta_{33}$. Using Eq. (3) together with an analytical relation for the out-of-plane polarization $P_{3}$, one can calculate the sensitivities $S_{11}^{\varepsilon}$ and $S_{22}^{\varepsilon}$ as a function of strains $u_{11}$ and $u_{22}$.

It is also instructive to compare the strain-mediated $\mathrm{MC}$ resulting from Eqs. (2) and (3) with the ME voltage coefficient ${ }^{30}$ of the same film-substrate hybrid. For the transverse ME coefficient $\alpha_{E 31}=\partial E_{3} / \partial H_{1}$ defining the electric field $E_{3}$ appearing in a ferroelectric capacitor with open-circuit electrical conditions under in-plane magnetic field $H_{1}$, the calculation gives

$$
\alpha_{E 31}=-\frac{Q_{12}\left(d_{111}^{\mathrm{m}}+d_{122}^{\mathrm{m}}\right)}{4\left(s_{11}+s_{12}\right)\left(a_{33}^{*}+3 a_{111} P_{3}^{2}+6 a_{1111} P_{3}^{4}\right) P_{3} \eta_{33}} .
$$

It can be seen that, in contrast to $\mathrm{MC}, \alpha_{E 31}$ is inversely proportional to the dielectric susceptibility $\eta_{33}$ and depends on the polarization $P_{3}$ in a different way. Accordingly, there is no direct proportionality between the $\mathrm{MC}$ and the ME voltage coefficient, contrary to the prediction made from general considerations in Ref. 34.

In the case of the $a$ a phase $\left(\left|P_{1}\right|=\left|P_{2}\right|, P_{3}=0\right)$ formed in a film subjected to an isotropic biaxial strain $\left(u_{11}=u_{22}=u_{m}\right.$, $\left.u_{12}=0\right)$, the strain sensitivities are given by the relation

$$
S_{11}^{\varepsilon}=S_{22}^{\varepsilon}=\frac{2 Q_{12}}{\left(s_{11}+s_{12}\right)}\left\{1-\frac{\left(Q_{11}+Q_{12}\right)\left[a_{13}^{*}+\left(2 a_{112}+a_{123}\right) P_{1}^{2}+3\left(a_{1112}+a_{1123}\right) P_{1}^{4}\right]}{Q_{12}\left[2 a_{11}^{*}+a_{12}^{*}+6\left(a_{111}+a_{112}\right) P_{1}^{2}+6\left(2 a_{1111}+2 a_{1112}+a_{1122}\right) P_{1}^{4}\right]}\right\} \eta_{33},
$$

where $\eta_{33}=\left[2 a_{3}^{*}+4 a_{13}^{*} P_{1}^{2}+2\left(2 a_{112}+a_{123}\right) P_{1}^{4}+4\left(a_{1112}+a_{1123}\right) P_{1}^{6}\right]^{-1}$ is the out-of-plane dielectric susceptibility of the $a a$ phase and $a_{1 j}^{*}$ are the renormalized thermodynamic coefficients introduced in Ref. 31 . If the measuring magnetic field $\delta \mathbf{H}$ does not induce shear strain $u_{12}$ in the film, Eq. (5) is sufficient to calculate the strain-mediated MC via Eq. (2). 

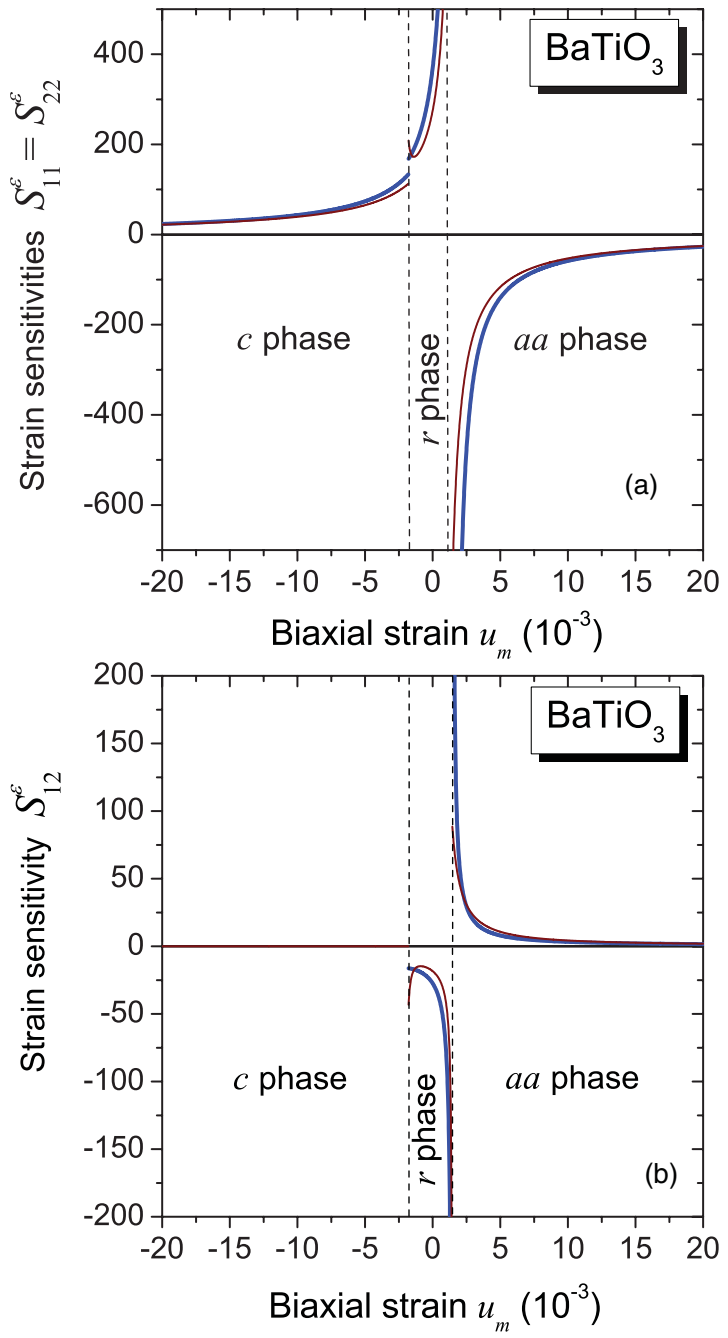

FIG. 2. (Color online) Sensitivities $S_{\alpha \beta}^{\varepsilon}=\partial \ln \varepsilon_{33} / \partial u_{\alpha \beta}$ of the out-of-plane permittivity $\varepsilon_{33}$ of single-domain $\mathrm{BaTiO}_{3}$ films to the (a) in-plane normal strains $u_{11}$ and $u_{22}$ and (b) shear strains $u_{12}$ as a function of the initial biaxial strain $u_{m}$ in the film. The thick and thin lines show sensitivities of the low- and high-frequency permittivities, respectively.

The numerical calculations were performed for $\mathrm{BaTiO}_{3}$ and PZT 50/50 films having initially an isotropic biaxial strain $u_{m}$. This strain state is typical of epitaxial films, ${ }^{35}$ corresponding, for instance, to (001)-oriented films grown on a (001)-oriented cubic or tetragonal bottom electrode or substrate $\left(u_{11}^{0}=\right.$ $\left.u_{22}^{0}=u_{m}, u_{12}^{0}=0\right)$. Small field-induced strains $u_{\alpha \beta}^{s}(\mathbf{H})$ were neglected in the calculations $\left(u_{\alpha \beta}=u_{\alpha \beta}^{0}\right)$. Figures 2 and 3 show the strain sensitivities $S_{\alpha \beta}^{\varepsilon}=S_{\alpha \beta}^{\varepsilon}\left(u_{m}\right)$ of the out-of-plane permittivity $\varepsilon_{33}$ as a function of the initial biaxial strain $u_{m}$ for $\mathrm{BaTiO}_{3}$ and PZT 50/50 films, respectively. Importantly, the sensitivities $S_{11}^{\varepsilon}=S_{22}^{\varepsilon}$ appear to be rather high even at large negative and positive misfit strains. For example, in $\mathrm{BaTiO}_{3}$ films $S_{\alpha \alpha}^{\varepsilon}\left(u_{m}=-1 \%\right) \cong 43$ and $S_{\alpha \alpha}^{\varepsilon}\left(u_{m}=+1 \%\right) \cong$ -58 , whereas in PZT 50/50 ones $S_{\alpha \alpha}^{\varepsilon}\left(u_{m}=-2 \%\right) \cong 23$ and $S_{\alpha \alpha}^{\varepsilon}\left(u_{m}=+2 \%\right) \cong-40$. Remarkably, $S_{\alpha \alpha}^{\varepsilon}$ increase drastically near the $r \leftrightarrow a a$ phase transition, where their magnitude exceeds 1000 .

At the same time, the sensitivity of $\varepsilon_{33}$ to shear strains differs from zero only in the $r$ and $a a$ phases (see Figs. 2 and 3). In
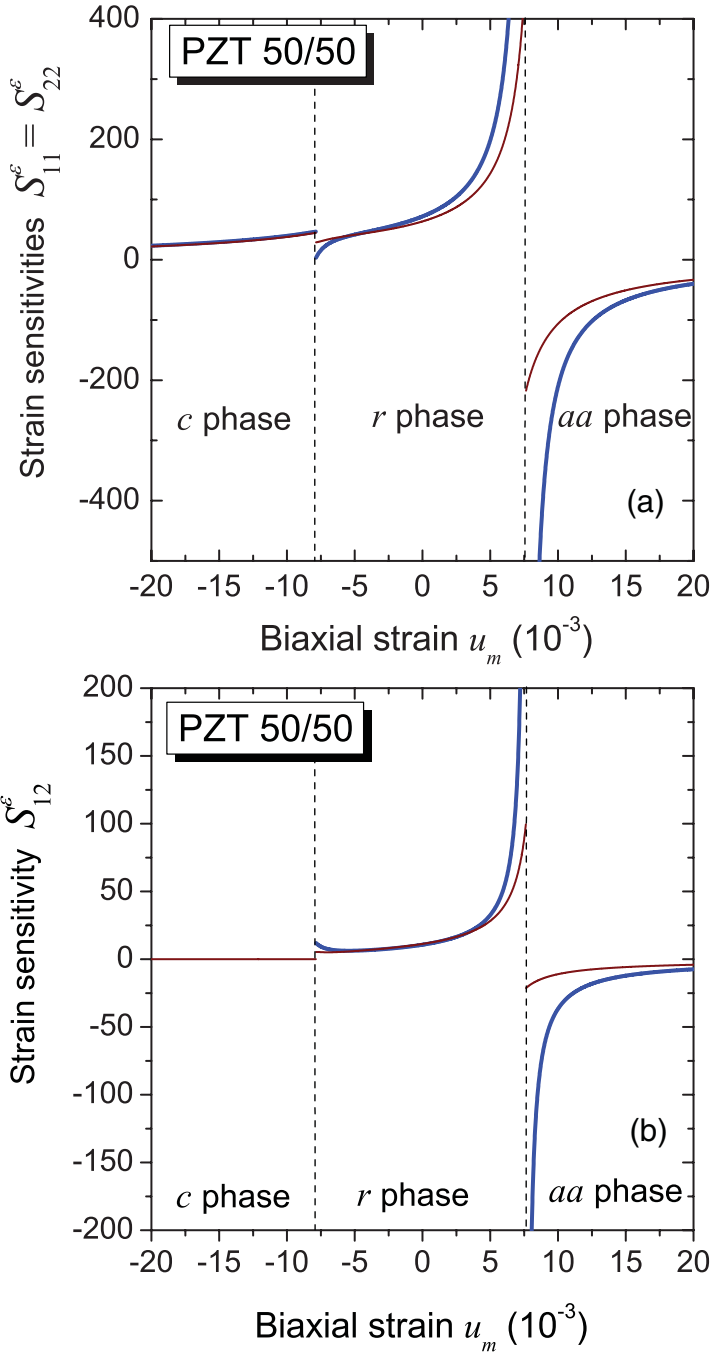

FIG. 3. (Color online) Sensitivities $S_{\alpha \beta}^{\varepsilon}=\partial \ln \varepsilon_{33} / \partial u_{33}$ of the outof-plane permittivity $\varepsilon_{33}$ of single-domain $\mathrm{Pb}\left(\mathrm{Zr}_{0.5} \mathrm{Ti}_{0.5}\right) \mathrm{O}_{3}$ films to the (a) in-plane normal strains $u_{11}$ and $u_{22}$ and (b) shear strains $u_{12}$ as a function of the initial biaxial strain $u_{m}$ in the film. The thick and thin lines show sensitivities of the low- and high-frequency permittivities, respectively.

$\mathrm{BaTiO}_{3}$ films, $S_{12}^{\varepsilon}$ is always more than one order of magnitude smaller than $S_{\alpha \alpha}^{\varepsilon}$. For PZT 50/50 films, the condition $S_{12}^{\varepsilon} \ll$ $S_{\alpha \alpha}^{\varepsilon}$ holds almost in the whole studied strain range, but in the vicinity of the $r \rightarrow c$ phase transition $S_{12}^{\varepsilon}$ becomes even larger than $S_{\alpha \alpha}^{\varepsilon}$.

To evaluate MC of a particular film-substrate hybrid, we have to specify the piezomagnetic coefficients $d_{j \alpha \beta}^{\mathrm{m}}(\mathbf{H})$ involved in Eq. (2). Since the coefficients $d_{3 \alpha \beta}^{\mathrm{m}}$ related to the measuring field $\delta H_{3}$ cannot be evaluated with sufficient precision (due to suppression of piezomagnetic response by the demagnetizing field), we focus on hybrids subjected to magnetic fields $\mathbf{H}$ and $\delta \mathbf{H}$ parallel to the film-substrate interface. In the most of magnetomechanical experiments, measuring and bias fields have the same direction and do not induce significant shear strains in a ferromagnetic sample. ${ }^{36-39}$ Therefore, we need to know only the longitudinal piezomagnetic coefficient characterizing the deformation $u^{\mathrm{s}}$ induced along the field direction and the transverse coefficient 
governing the deformation $u_{\perp}^{\mathrm{s}}$ in the direction orthogonal to $\delta \mathbf{H}$. Denoting these coefficients by $d_{\|}^{\mathrm{m}}$ and $d_{\perp}^{\mathrm{m}}$, respectively, we obtain

$$
\mathrm{MC}=\left[S_{11}^{\varepsilon}\left(d_{\|}^{\mathrm{m}}+d_{\perp}^{\mathrm{m}}\right)-\frac{1}{2} S_{12}^{\varepsilon}\left(d_{\|}^{\mathrm{m}}-d_{\perp}^{\mathrm{m}}\right) \sin 2 \varphi\right] \delta H,
$$

where $\varphi$ is the angle between the magnetic field $\delta \mathbf{H}$ and the [100] crystallographic axis of the film. Among bulk ferromagnets, the highest longitudinal coefficients are displayed by the FeBSiC alloy $\left(d_{\|}^{\mathrm{m}} \approx 50 \mathrm{~nm} / \mathrm{A}\right),{ }^{39}$ grain-oriented Terfenol-D polycrystal $\left(d_{\|}^{\mathrm{m}} \approx 30 \mathrm{~nm} / \mathrm{A}\right),{ }^{36}$ and FeGa and Terfenol-D single crystals $\left(d_{\| !}^{\mathrm{m}} \approx 20 \mathrm{~nm} / \mathrm{A}\right) .{ }^{37,38}$ The transverse coefficients of these materials may be estimated from the condition of volume conservation, which gives $d_{\perp}^{\mathrm{m}}=-d_{\|}^{\mathrm{m}} / 2$. It can be shown that this relation holds for the macroscopic deformations resulting from magnetization reorientations in polydomain crystals of cubic ferromagnets, where magnetic domains have randomly oriented magnetizations of fixed magnitude. ${ }^{30}$ The experimental measurements show that magnetic-field-induced deformations conserve volume to first order in samples of cubic symmetry. ${ }^{40}$

Substituting the numerical values of $d_{\|}^{\mathrm{m}}$ and $d_{\perp}^{\mathrm{m}}$ into Eq. (6), we find that the magnetocapacitive coefficient $\mathrm{MC} / \delta H$ of hybrids comprising $\mathrm{BaTiO}_{3}$ or PZT 50/50 films can easily reach $\sim 10^{-4} \mathrm{Oe}^{-1} .^{41}$ Remarkably, this value exceeds by two orders of magnitude the coefficients $\mathrm{MC} / \delta H \sim 10^{-6} \mathrm{Oe}^{-1}$ characteristic of multiferroic heterostructures grown on passive substrates. ${ }^{15,17}$ Moreover, the dependences $S_{\alpha \beta}^{\varepsilon}\left(u_{m}\right)$ plotted in Figs. 2 and 3 demonstrate that, near the $r \leftrightarrow a a$ phase transition, the strain-mediated response of the discussed multiferroic hybrids reaches giant values $\sim 10^{-3} \mathrm{Oe}^{-1}$. As a result, the MC of hybrids including Terfenol-D may exceed $100 \%$ already at $\delta H \approx 600 \mathrm{Oe}$, which is two orders of magnitude higher than $\mathrm{MC} \sim 1 \%$ displayed by multiferroic heterostructures grown on passive substrates. ${ }^{15,17}$

It should be emphasized that the calculated MC corresponds to frequencies $v$ of the measuring ac electric field, which are well below the frequency $v_{\text {res }}$ of the film mechanical resonance. Indeed, the out-of-plane strain $u_{33}$ in the ferroelectric film was determined from the condition of zero stress $\sigma_{33}$ here, which implies that $u_{33}$ oscillates with the frequency $v$ owing to periodic piezoelectric deformations accompanying field-induced polarization changes. These deformations fully develop at frequencies $v \leqslant v_{\text {res }}$ but become suppressed above $\nu_{\text {res }}$, which reduces the film susceptibility $\eta_{33}$ and affects the MC. By setting $u_{33}$ fixed at the initial value existing in the absence of an electric field, we calculated the high-frequency susceptibility $\eta_{33}^{\text {c }}$ and MC. For the $c$ phase, $\eta_{33}^{\mathrm{c}}$ was found to be $\eta_{33}^{\mathrm{c}}=$ $\left(2 a_{3}^{*}+12 a_{33}^{* \mathrm{c}} P_{3}^{2}+30 a_{111} P_{3}^{4}+56 a_{1111} P_{3}^{6}\right)^{-1}$, where $a_{33}^{* \mathrm{c}}=$ $a_{11}+Q_{12}^{2} /\left[3\left(s_{11}+s_{12}\right)\right]+\left[\left(Q_{11}^{2}+2 Q_{12}^{2}\right) s_{11}+Q_{11}\left(Q_{11}-\right.\right.$ $\left.\left.4 Q_{12}\right) s_{12}\right] /\left[3\left(s_{11}-s_{12}\right)\left(s_{11}+2 s_{12}\right)\right]$ is the "clamped" second-order thermodynamic coefficient. Hence, the strain sensitivities of high-frequency permittivity can be calculated from the relation

$$
\begin{aligned}
S_{11}^{\varepsilon \mathrm{c}}= & S_{22}^{\varepsilon \mathrm{c}}=\frac{2 Q_{12}}{\left(s_{11}+s_{12}\right)} \\
& \times\left(1-3 \frac{a_{33}^{* \mathrm{c}}+5 a_{111} P_{3}^{2}+14 a_{1111} P_{3}^{4}}{a_{33}^{*}+3 a_{111} P_{3}^{2}+6 a_{1111} P_{3}^{4}}\right) \eta_{33}^{\mathrm{c}} .
\end{aligned}
$$

The numerical calculations showed that, in the $c$ phase, $S_{\alpha \alpha}^{\varepsilon \mathrm{c}}$ are only slightly smaller than the strain sensitivities $S_{\alpha \alpha}^{\varepsilon}$ of low-frequency permittivity. Near the $r \leftrightarrow a a$ phase transition, however, the discussed sensitivities become significantly reduced at $v>v_{\text {res }}$ (see Figs. 2 and 3). Nevertheless, there are wide strain ranges, where the high-frequency $\mathrm{MC}$ is comparable to the low-frequency one.

\section{CONCLUDING REMARKS}

In this paper, we reported a theoretical calculation of the strain-mediated magnetocapacitance of ferroelectricferromagnetic heterostructures. To evaluate this magnetocapacitance, we employed the nonlinear thermodynamic theory because the linear theory does not predict any dependence of electric permittivity on lattice strains. The calculation revealed several important features of the strain-mediated magnetocapacitance, such as nonmonotonic variation with initial lattice strains existing in the ferroelectric film, drastic increase at the strain-induced $r \leftrightarrow a a$ phase transition, and significant dependence on the frequency of measuring electric field, which appears in the vicinity of this transition near the frequency of the film piezoelectric resonance. We showed that the magnetodielectric effect exhibited by hybrids combining ferroelectric thin films with active ferromagnetic substrates is several orders of magnitude higher than weak effects displayed by multiferroic heterostructures grown on passive substrates.

Remarkably, the strain-mediated magnetocapacitance appears to be significant even in the absence of any out-of-plane polarization $P_{3}$ in the ferroelectric film. This feature represents a striking contrast between the magnetocapacitance and the magnetoelectric voltage coefficient, which becomes negligible at $P_{3}=0$ in the conventional parallel-plate capacitor geometry. ${ }^{29,30}$ Therefore, multiferroic hybrids comprising ferroelectric films subjected to tensile in-plane strains should not be ignored in experimental studies of magnetoelectric phenomena.

Finally, it should be emphasized that the predicted giant magnetodielectric effect has potential applications in advanced electronic devices. In particular, it can be exploited in magnetic-field-controlled ferroelectric capacitors. These magnetovaractors may be implemented in electronic oscillators, where the oscillation frequency is tuned by a dc magnetic field remotely (via the change of capacitance) in contrast to conventional voltage-controlled oscillators. Magnetovaractors can also be used in parametric oscillators, where the oscillation is driven by an ac magnetic field changing the capacitance periodically.

${ }^{3}$ R. Ramesh and N. A. Spaldin, Nature Mater. 6, 21 (2007).

${ }^{4}$ C. A. F. Vaz, J. Hoffman, C. H. Ahn, and R. Ramesh, Adv. Mater. 22, 2900 (2010).

\footnotetext{
${ }^{1}$ N. A. Spaldin and M. Fiebig, Science 309, 391 (2005).

${ }^{2}$ W. Eerenstein, N. D. Mathur, and J. Scott, Nature (London) 44, 759 (2006).
} 
${ }^{5}$ W. Eerenstein, M. Wiora, J. L. Prieto, J. F. Scott, and N. V. Mathur, Nature Mater. 6, 348 (2007).

${ }^{6}$ S. Dong, J. F. Li, and D. Viehland, Appl. Phys. Lett. 83, 2265 (2003).

${ }^{7}$ G. Srinivasan, A. S. Tatarenko, and M. I. Bichurin, Electron. Lett. 41, 10 (2005).

${ }^{8}$ C. Pettiford, S. Dasgupta, J. Lou, S. D. Yoon, and N. X. Sun, IEEE Trans. Magn. 43, 3343 (2007).

${ }^{9}$ M. Vopsaroiu, J. Blackburn, A. Muniz-Piniella, and M. G. Cain, J. Appl. Phys. 103, 07F506 (2008).

${ }^{10}$ N. A. Pertsev and H. Kohlstedt, Appl. Phys. Lett. 95, 163503 (2009).

${ }^{11}$ N. A. Pertsev and H. Kohlstedt, Nanotechnology 21, 475202 (2010).

${ }^{12}$ C. Israel, N. D. Mathur, and J. F. Scott, Nature Mater. 7, 93 (2008).

${ }^{13}$ H. Zheng, J. Wang, S. E. Lofland, Z. Ma, L. Mohaddes-Ardabili, T. Zhao, L. Salamanca-Riba, S. R. Shinde, S. B. Ogale, F. Bai,

D. Viehland, Y. Jia, D. G. Schlom, M. Wuttig, A. Roytburd, and R. Ramesh, Science 303, 661 (2004).

${ }^{14}$ M. P. Singh, W. Prellier, C. Simon, and B. Raveau, Appl. Phys. Lett. 87, 022505 (2005).

${ }^{15}$ R. Chaudhuri, P. Mandal, S. B. Krupanidhi, and A. Sundaresan, Solid State Commun. 148, 566 (2008).

${ }^{16}$ J. H. Park, H. M. Jang, H. S. Kim, C. G. Park, and S. G. Lee, Appl. Phys. Lett. 92, 062908 (2008).

${ }^{17}$ Y. Chen, G. Wang, S. Zhang, X. Lei, J. Zhu, X. Tang, Y. Wang, and X. Dong, Appl. Phys. Lett. 98, 052910 (2011).

${ }^{18}$ M. A. Zurbuchen, T. Wu, S. Saha, J. Mitchell, and S. K. Streiffer, Appl. Phys. Lett. 87, 232908 (2005).

${ }^{19}$ T. Wu, M. A. Zurbuchen, S. Saha, R.-V. Wang, S. K. Streiffer, and J. F. Mitchell, Phys. Rev. B 73, 134416 (2006).

${ }^{20}$ C. Thiele, K. Dörr, O. Bilani, J. Rödel, and L. Schultz, Phys. Rev. B 75, 054408 (2007).

${ }^{21}$ A. Brandlmaier, S. Geprägs, M. Weiler, A. Boger, M. Opel, H. Huebl, C. Bihler, M. S. Brandt, B. Botters, D. Grundler, R. Gross, and S. T. B. Goennenwein, Phys. Rev. B 77, 104445 (2008).

${ }^{22}$ J. Lou, M. Liu, D. Reed, Y. Ren, and N. X. Sun, Adv. Mater. 21, 4711 (2009).

${ }^{23}$ T. Wu, A. Bur, P. Zhao, K. P. Mohanchandra, K. Wong, K. L. Wang, C. S. Lynch, and G. P. Carman, Appl. Phys. Lett. 98, 012504 (2011).
${ }^{24}$ G. Catalan, Appl. Phys. Lett. 88, 102902 (2006).

${ }^{25}$ J. F. Scott, J. Mater. Res. 22, 2053 (2007).

${ }^{26}$ C. W. Nan, M. Li, and J. H. Huang, Phys. Rev. B 63, 144415 (2001).

${ }^{27}$ M. I. Bichurin, V. M. Petrov, and G. Srinivasan, Phys. Rev. B 68, 054402 (2003).

${ }^{28}$ V. M. Petrov, G. Srinivasan, M. I. Bichurin, and A. Gupta, Phys. Rev. B 75, 224407 (2007).

${ }^{29}$ N. A. Pertsev, H. Kohlstedt, and B. Dkhil, Phys. Rev. B 80, 054102 (2009).

${ }^{30}$ V. G. Kukhar, N. A. Pertsev, and A. L. Kholkin, Nanotechnology 21, 265701 (2010).

${ }^{31}$ N. A. Pertsev, A. G. Zembilgotov, and A. K. Tagantsev, Phys. Rev. Lett. 80, 1988 (1998).

${ }^{32}$ A. G. Zembilgotov, N. A. Pertsev, U. Böttger, and R. Waser, Appl. Phys. Lett. 86, 052903 (2005).

${ }^{33}$ N. A. Pertsev, V. G. Kukhar, H. Kohlstedt, and R. Waser, Phys. Rev. B 67, 054107 (2003).

${ }^{34}$ H. M. Jang, J. H. Park, S. Ryu, and S. R. Shannigrahi, Appl. Phys. Lett. 93, 252904 (2008).

${ }^{35}$ D. G. Schlom, L.-Q. Chen, C.-B. Eom, K. M. Rabe, S. K. Streiffer, and J.-M. Triscone, Annu. Rev. Mater. Res. 37, 589 (2007).

${ }^{36}$ M. B. Moffett, A. E. Clark, M. Wun-Fogle, J. Linberg, J. P. Teter, and E. A. McLaughin, J. Acoust. Soc. Am. 89, 1448 (1991).

${ }^{37}$ B. W. Wang, S. C. Busbridge, Y. X. Li, G. H. Wu, and A. R. Piercy, J. Magn. Magn. Mater. 218, 198 (2000).

${ }^{38}$ E. Clark, M. Wun-Fogle, J. B. Restorff, T. A. Lograsso, and J. R. Cullen, IEEE Trans. Magn. 37, 2678 (2001).

${ }^{39}$ S. X. Dong, J. Zhai, J. Li, and D. Viehland, Appl. Phys. Lett. 89, 252904 (2006)

${ }^{40}$ R. C. O'Handley, S. J. Murray, M. Marioni, H. Nembach, and S. M. Allen, J. Appl. Phys. 87, 4712 (2000).

${ }^{41}$ It should be noted that the fabrication of a single-crystalline or highly textured ferroelectric film directly on a ferromagnetic substrate may be unfeasible. However, this can be achieved by incorporating an appropriate buffer layer into the heterostructure, which ensures epitaxial growth of a ferroelectric film on the bottom electrode. Another suitable technology includes the film growth on a thin passive substrate, which is cemented to a magnetostrictive actuator afterward. 